\title{
Scleral Concave Pool Trabeculectomy Combined Phacoemulsification in Primary Open-Angle Glaucoma with Cataract.
}

\section{Xiangxiang Ye}

Guangdong Hospital of Traditional Chinese Medicine

\section{Yongjun Qi ( $\nabla$ qiyjyk@163.com )}

Guangdong Hospital of Traditional Chinese Medicine https://orcid.org/0000-0001-7813-922X

Jianhua Deng

Guangdong Hospital of Traditional Chinese Medicine

\section{Yang Yang}

Guangdong Hospital of Traditional Chinese Medicine

\section{Ting Mo}

Guangdong Hospital of Traditional Chinese Medicine

\section{Mao Xu}

Guangdong Hospital of Traditional Chinese Medicine

\section{Wanjun Liu}

Guangdong Hospital of Traditional Chinese Medicine

\section{Research article}

Keywords: Primary open-angle glaucoma, scleral concave pool trabeculectomy, cataract, phacoemulsification, intraocular pressure

Posted Date: April 14th, 2020

DOI: https://doi.org/10.21203/rs.2.19458/v2

License: (c) (1) This work is licensed under a Creative Commons Attribution 4.0 International License. Read Full License

Version of Record: A version of this preprint was published at BMC Ophthalmology on June 9th, 2020. See the published version at https://doi.org/10.1186/s12886-020-01500-2. 


\section{Abstract}

Background: To investigate the safety and efficacy of scleral concave pool trabeculectomy (SCPT) combined phacoemulsification for eyes with coexisting cataract and primary open-angle glaucoma (POAG).

Methods: This was a retrospective, controlled, interventional case series. Thirty patients (30 eyes) were diagnosed with coexisting cataract and POAG between May 2015 and April 2018. Fourteen eyes underwent SCPT combined phacoemulsification were set as the study group, and 16 eyes received conventional phacotrabeculectomy were set as the control group. All patients were followed up for at least 6 months. The preoperative to postoperative changes in IOP, glaucoma medication requirements, BCVA, blebs functions, and adverse events were recorded.

Results: The groups were matched for baseline age, BCVA, IOP and types of IOP-lowering medications (all $P>0.05)$. At 6-month visit, there were no significant difference between control and study group in the improvement of BCVA $(0.22 \pm 0.24$ versus $0.18 \pm 0.26, P=0.718)$, reduction of IOP $(-11.21 \pm 8.61 \mathrm{mmHg}$ versus $-9.19 \pm 9.18 \mathrm{mmHg}, \mathrm{P}=0.540$ ) and the number of eyes that needed IOP-lowering medications (2 versus $3, P=0.743)$. At the last visit, the rate of forming functioning blebs was significantly different between the study and control groups, $(92.9 \%$ versus $68.7 \%$ respectively, $\mathrm{P}=0.007)$. In the study group, 5 eyes developed hypotony $₫$ and 1 eye showed limited choroidal detachment, whereas in the control group 1 eye developed malignant glaucoma. All adverse events were successfully managed.

Conclusion: The SCPT combined phacoemulsification is a safe and effective alternative to conventional phacotrabeculectomy for patients with POAG and visually significant cataract.

\section{Background}

Primary open-angle glaucoma (POAG) is one of the major causes of blindness for elderly people who often suffer from coexisting cataract ${ }^{[1]}$. In such patients, combined phacoemulsification and trabeculectomy (phacotrabeculectomy) can treat coexisting visually significant cataract and advance glaucoma at the same time ${ }^{[2]}$ which is the preferred surgical management in Chinese elderly patients. Fibroblast proliferation and collagen deposition at filter channel is the most common reason for trabeculectomy failure ${ }^{[3-5]}$. It is widely accepted that the application of adjunctive antifibrotic agents such as 5-fluorouracil (5-FU) and mitomycin $\mathrm{C}(\mathrm{MMC})$ can enhance the success rate of filtration surgery $[6,7]$. Unfortunately, filtering surgery still fails in some cases, and has serious adverse effects ${ }^{[8]}$. In addition, during a certain period of time, there was not enough supply of 5-FU and MMC drugs in China because many domestic companies stopped producing them. Therefore, it sparks an emergency requirement for the improvement of trabeculectomy technique.

Improvement of techniques, such as deep sclerectomy (DS) and $\mathrm{CO}_{2}$ laser-assisted sclerectomy surgery (CLASS) has reduced the complication rates in treating open angle glaucoma ${ }^{[9-11]}$. Contrary to 
trabeculectomy, DS, which does not require penetrating the anterior chamber, has been reported to improve the safety of glaucoma surgery with high success rate, but it requires considerable technical skills to preserve the integrity of the trabeculo-Descemet's membrane (TDM) ${ }^{[9,12]}$. Recently, a newly developed technique, CLASS, which performs deep sclerectomy down to the TDM by $\mathrm{CO}_{2}$ laser application on the scleral bed after a half thickness scleral flap is created, has been reported as a feasible and apparently safe procedure ${ }^{[11,13]}$. However, the IOPtiMate system used in the CLASS procedure is too expensive for its wide spread application in developing countries such as China. Furthermore, CLASS technique is a non-penetrating technique which requires a long learning curve.

To our knowledge, a reservoir in the CLASS procedure allowing the percolated aqueous accumulation may help strike a balance between the production and outflow of aqueous humor, which has the advantages of keeping the filtering path unobstructed and reduces the postoperative complication of filtering surgery. Therefore『we developed a penetrating surgery technique to add a scleral concave pool manually in the trabeculectomy procedure which needs shorter learning curve than non-penetrating technique. In our retrospective study, we aimed to investigate the safety and efficacy of scleral concave pool trabeculectomy surgery (SCPT), performed in conjunction with phacoemulsification, for treating eyes with coexisting cataract and POAG.

\section{Methods}

We reviewed the operations performed by one surgeon (Yongjun Qi) at the Guangdong Hospital of Traditional Chinese Medicine, Zhuhai, China between May 2015 and April 2018. All patients considered in this study had coexisting cataract and primary open angle glaucoma. Fourteen eyes (14 patients) underwent SCPT combined phacoemulsification were set as the study group, and 16 eyes (16 patients) received conventional phacotrabeculectomy were set as the control group. Patients were excluded from this study if any of the following conditions exists: gonioscopically narrow or closed angle, history of any vitreo-retinal surgery, fundus diseases (such as age-related macular degeneration, diabetic retinopathy, and retinal detachment), inflammatory eye disorders, or any other potential cause of vision loss. All patients received regular follow-up examinations for at least 6 months.

\section{Intervention}

All procedures followed the tenets of the Declaration of Helsinki. This study obtained approval from the ethical committee of Guangdong Hospital of Traditional Chinese Medicine, Zhuhai, China. Written informed consent was obtained after explanation of the nature of the procedure.

In both groups, all surgery procedures were performed under peribulbar anesthesia. Before filtration surgeries, phacoemulsification and intraocular lens implantation were performed. A fornix-based conjunctival flap was dissected in the superior quadrant, and the sclera was exposed. A $4 \times 4 \mathrm{~mm}$ limbusbased scleral flap was then created. For the SCPT procedure, a reservoir measuring about $3 \times 2 \mathrm{~mm}$ was created over the posterior scleral bed by a surgical blade (Fig. 1). The deeper sclera and the root of 
Schlemm canal were excised through a convex groove. The superficial scleral flap was closed with 10-0 nylon sutures, and the conjunctival wound was sutured with 10-0 nylon sutures. For the conventional phacotrabeculectomy, no reservoir was further performed. The superficial scleral flap and the conjunctival wound were sutured straightly through the same way with 10-0 nylon sutures. Postoperative management in both groups included overnight patching, topical levofloxacin $5 \mathrm{mg} / \mathrm{ml}$ and dexamethasone $1 \mathrm{mg} / \mathrm{ml}$ for 4 weeks. Glaucoma medications were administered if necessary.

Postoperatively, all eyes received topical tobramycin and dexamethasone eye drops every 4 hours for 1 week and tobramycin and dexamethasone eye ointment every night for 1 week, with gradual tapering over the next 4 weeks. Glaucoma medications were administered if IOP $>21 \mathrm{mmHg}$. An additional trabeculectomy would be required, if the IOP remained uncontrolled even after maximally-tolerated glaucoma drugs, systemic anti-inflammatory or bleb manipulation were offered.

\section{Outcome Measures}

Demographics, systemic diseases, ophthalmic history, topical and systemic medications before the operation were collected. Ocular examinations conducted at baseline and at each follow-up visit (1 day, 1 week, 1 month and 6 months) including best corrected visual acuity (BCVA) with Snellen chart, Goldmann applanation tonometry, slit-lamp examination, gonioscopy, and fundus examination were

reviewed. Filtering blebs were categorized as 4 types according to the Kronfeld's classification ${ }^{[14]}$. In brief, thin and polycystic blebs with transconjunctival flow of fluid were classified as Type I bleb, other flatter, thicker, and more diffuse blebs with a relatively vascular appearance as Type II bleb, failed bleb in which conjunctiva is scarred to underlying episclera as Type III bleb, and encapsulated bleb with characteristic vascular, dome-shaped, cyst-like appearance as Type IV bleb. Procedure related complications and number of IOP lowering medications administrated postoperatively were recorded. In the current study, completed success meant postoperative IOP remained stable and was less than $21 \mathrm{mmHg}$ without glaucoma medication, while qualified success meant postoperative IOP remained stable and was less than $21 \mathrm{mmHg}$ with glaucoma medication.

\section{Data analysis}

Data were first tested for normality using Sample K-S test. Variable confirming to normal distribution were summarized as means \pm standard deviation (SD) except when stated otherwise. Comparisons of normally distributed variables between the groups were conducted using independent samples $t$ test, or with nonparametric test if variables are not normally distributed. Changes in BCVA and IOP from baseline to four follow-up visits between the 2 groups were examined using a repeated measure ANOVA. The number of functioning blebs between the two groups at each visit was compared using Mann-Whitney $U$ test. Statistical analysis was performed using SPSS Version 16.0 (SPSS 16.0, Inc., Chicago, IL). Significance was determined as $\mathrm{P}<0.05$ at two tails.

\section{Results}


As shown in Table 1, there was no significant difference in the mean baseline age, gender, BCVA, IOP and number of IOP-lowering medications used between the two groups (all $P>0.05$ ). Compared to baseline, postoperative BCVA significantly improved at 1-week, 1-month and 6-month visit in both groups (all $\mathrm{P}<0.05)$, but the magnitude of visual improvement was comparable between both groups at each visit (all $\mathrm{P}>0.05$ ) (Fig. 2).

\section{Intraocular Pressure and Number of Medications}

Figure 3 shows the IOP changes in the two groups during the follow-up. In both groups, the IOP significantly decreased from baseline at each visit. Mean reduction of IOP was $-11.21 \pm 8.61 \mathrm{mmHg}$ in the study group and $-9.19 \pm 9.18 \mathrm{mmHg}$ in the control group at the last visit. There was no significant difference in the magnitude of IOP reduction between the 2 groups at the each visit (all P>0.05). During the follow-up, the number of eyes did not require any IOP-lowering medication was 12 and 11 in the study and control groups, respectively. The number of eyes received ophthalmic solution to lower the IOP was 2 and 3 in the study and control groups, respectively. In addition, no eye needs postoperative bleb manipulation in the study group, whereas 2 eyes require postoperative bleb manipulation to help control the IOP in the control group.

\section{Blebs}

At the 1-month visit, all eyes showed functioning blebs in both groups. At the last visit, 13 eyes had functioning blebs, and only 1 eye had a Type III bleb in the study group, whereas 5 eyes had failed blebs (3 Type III and 2 Type IV) in the control groups. At the last visit, rate of forming functioning blebs was $92.9 \%$ and $68.7 \%$ in the study and control groups respectively, with a significant inter-group difference $(P=0.007)$.

\section{Complications}

As for adverse events related to the procedure and their management, in the study group, 4 eyes that developed hypotony with iris adhesion at 3 days post operation were successfully managed with mydriasis of atropine and tensioning the transconjunctival scleral flap sutures. 1 eye that showed hypotony and limited choroidal detachment required systemic anti-inflammatory treatment, mydriasis of atropine and tensioning the transconjunctival scleral flap sutures. In the control group, 1 eye that developed malignant glaucoma at 2 weeks post operation was successfully managed with Nd:YAG laser, mydriasis of atropine, $250 \mathrm{ml}$ of mannitol $20 \%$ intravenous infusion, and systemic anti-inflammatory treatment.

\section{Success Rate}

At the end of the 6-month period, SCPT combined phacoemulsification had a higher qualified successful rate than that of the control group $(100.00 \%$ vs. $87.5 \%, \mathrm{P}<0.01)$. Completed success rate was significantly higher in the study group after 6-month intervention (study: $85.70 \%$, control: 68.75\%, $\mathrm{P}=0.027$ ), with more patients underwent SCPT combined phacoemulsification meeting the complete success criteria. 
Postoperative IOP control was still larger than $21 \mathrm{mmHg}$ in 2 eyes of the control groups $(24 \mathrm{mmHg}$ and $25 \mathrm{mmHg}$, respectively) even after 3 kinds of glaucoma drugs and bleb manipulation were provided.

\section{Discussion}

In the current study, SCPT combined phacoemulsification was comparable to conventional phacotrabeculectomy in terms of reducing IOP and the number of medications over the first 6-month period but has higher rate of forming functional blebs. Patient undergoing SCPT combined phacoemulsification may be easier to develop hypotony in the early stage, but it could recover after some appropriate management. To the best of our knowledge, we are one of the few in making this new improvement in filtering surgery and comparing its safety and efficacy to phacotrabeculectomy, which still needs further improvement due to its postoperative complications ${ }^{[15]}$

Lowering of IOP remains the main therapeutic strategy in the treatment of glaucoma, and trabeculectomy is the most widely applied filtration surgical procedure for achieving a target IOP, with up to $90 \%$ of long term success to maintain vision-related quality of life ${ }^{[16-18]}$. The main purpose of filtration surgery is to lower the IOP by creating a path for more efficient drainage of the aqueous humor from the anterior chamber to the subconjunctival space. Therefore, maintaining the filtering path unobstructed is of critical importance. However冈trabeculectomies are often complicated with wound healing, postoperative fibrosis, and production of inflammatory mediators which cause bleb failure and subsequent closure of the filtering route ${ }^{[5,19]}$. 5-minute exposures of Mitomycin $\mathrm{C}$ during the procedure was insufficient in preventing the closure of filtering route and the failures of long term control of IOP due to the subconjunctival and scleral fibroblast proliferation ${ }^{[20]}$.

In the current study, both procedures had similar effects on reducing IOP and the number of medications used over the 6-month follow-up period, but both the complete (85.70\% vs. 68.75\%, $\mathrm{P}=0.027)$ and qualified success rate $(100.00 \%$ vs. $87.50 \%, P<0.01)$ were significantly higher in the study group compared to the control group. In terms of blebs, the rate of forming functioning blebs in the study group was also significantly higher than that in the control group (92.9\% vs. 68.7\%, $P=0.007)$. All these results may suggest a better efficacy for the long term reduction in IOP during the procedure of SCPT combined phacoemulsification compared to conventional phacotrabeculectomy, but further work is needed to confirm the increased efficacy by reviewing long term follow up.

SCPT, as a conventional filtering surgery, have some unavoidable early complications such as postoperative anterior chamber inflammation ${ }^{21]}$, bleb leaks ${ }^{[22]}$, intraocular hypotony ${ }^{[23]}$, and choroidal effusion etc ${ }^{[24]}$ all may threaten the VA. In the current study, both surgery procedures had similar effects on the improvement of postoperative VA, even though the postoperative complications were different between them. At 2 weeks post conventional phacotrabeculectomy, 1 eye developed malignant glaucoma, which was successfully managed with some appropriate treatment. Post SCPT combined phacoemulsification, 5 eyes developed hypotony, but all of them were successfully managed by appropriate intervention. Overall, this modification of conventional filtering surgery seemed to be as safe 
as the conventional phacotrabeculectomy at least over the early 6-month period, but its long-term safety still warrants investigation by further studies.

The CLASS procedure, which showed a similar qualified success rate and reduction in medications compared to conventional trabeculectomy, may be an alternative to trabeculectomy when considering the postoperative complications $[11,13,25,26]$. During the CLASS procedure, a reservoir is formed by using the $\mathrm{CO}_{2}$ laser to ablate the scleral tissue ${ }^{[11]}$. To our knowledge, a reservoir allowing the percolated aqueous accumulation may have the advantage of keeping the filtering path unobstructed by the following pathways: 1) Filling the bleb with aqueous humor all the time which then help maintain the tension of bleb; 2 ) Help regulate the flow of liquids so that when the flow speed is low in the drainage, the accumulated aqueous humor in the reservoir can offer a replenishment of liquid; 3 ) Keeping the path filled with liquid flow can help wash away inflammatory factors. The $\mathrm{CO} 2$ laser, which can be largely absorbed by water, has little direct effect on the angle tissue when the aqueous starts to percolate ${ }^{[11,13]}$, However, the IOPtiMate system used in the CLASS procedure is too expensive for its wide spread application in developing countries such as China. Thus, by adopting the advantage of the CLASS procedure, we decided to manually create the reservoir by surgical blade during the filtering surgery. This procedure needs considerable technical skills, but all the surgery procedures were conducted by one experienced surgeon which could help reduce bias of the result. according to previous studies, CLASS, as a nonpenetrating filtration surgery, may be an alternative to trabeculectomy, especially at the earlier glaucoma stage, considering its more attractive complications profile. However, CLASS is less effective than trabeculectomy and cannot be applied to many conditions such as the primary narrow and occludable angles or secondary glaucoma with the presence of peripheral anterior synechia ${ }^{[11,13]}$. However, according to the result of this study, by adopting the advantages of CLASS to penetration filtration surgery, SCPT combined phacoemulsification has comparable or even superior efficiency in reducing the IOP than conventional phacotrabeculectomy.

There were some limitations in this study. Firstly, the sample size was relative small, but it may be unsuited to conduct this procedure for too many patients at this stage since it is a new, untested technique. Furthermore, the follow-up time was relative short, but its short-term efficacy and safety which was the main purpose of this study could provide some positive implication for its long-term investigation. Finally, the procedure of SCPT may require considerable technical skills to form a reservoir by surgical blade, but it could be conquered by more practice.

\section{Conclusions}

In conclusion, by applying the advantage of CLASS to conventional trabeculectomy, SCPT combined phacoemulsification showed a similar efficacy in reducing IOP compared to phacotrabeculectomy, but it increased the success rate of forming functional blebs which provides a better implication for the longterm reduction in IOP. Eyes in the early post-procedure period may have a tendency to develop hypotony, but it can be successfully managed or theoretically recover after a longer follow-up time. Due to the 
recentness of the procedure's invention, the indication of SCPT combined phacoemulsification and the longevity of post-operative IOP control remains unknown. Therefore, the revision of our new trabeculectomy technique is still necessary. In addition, we suggest that further randomized prospective studies should compare this new surgery procedure to modern filtration surgery in many conditions, including primary open angle glaucoma, primary angle closure glaucoma or secondary glaucoma etc., with lager sample size and longer follow-up time to monitor its safety, long-term efficacy and indications.

\section{Declarations}

\section{- Ethics approval and consent to participate}

All procedures followed the tenets of the Declaration of Helsinki. This study obtained approval from the ethical committee of Guangdong Hospital of Traditional Chinese Medicine, Zhuhai, China. Written informed consent was obtained after explanation of the nature of the procedure.

\section{- Consent to publish}

All the authors have made special contribution to this manuscript and consent to publish it in the journal of "BMC Ophthalmology".

\section{- Availability of data and materials}

Data and material can be found at the following URL: https://pan.baidu.com/s/1VpZbSs7V9hDUm87Rbeq2g.

\section{- Competing interests}

The authors declare that they have no competing interests.

\section{- Funding}

This work was supported by no grants.

\section{- Authors' Contributions}

Involved in the design of the study (XYX, YQ); conduct of the study ( $X Y, Y Q, J D)$; collection, management, analysis of the data $(X Y, J D, Y Y, T M, M X, W L)$; preparation of the manuscript $(X Y, J D)$; and critical revision of the manuscript (YQ). All authors read and approved the final manuscript.

\section{- Acknowledgements}

Not applicable

\section{Abbreviations}


SCPT: scleral concave pool trabeculectomy; POAG: primary open angle glaucoma; 5-FU: 5-fluorouracil; MMC: mitomycin C; DS: deep sclerectomy; CLASS: $\mathrm{CO}_{2}$ laser-assisted sclerectomy surgery; TDM: trabeculo-Descemet's membrane; BCVA: best corrected visual acuity; IOP: intraocular pressure.

\section{References}

[1] Zetterberg M. Age-related eye disease and gender. Maturitas. 2016. 83: 19-26.

[2] Rao HL, Maheshwari R, Senthil S, Prasad KK, Garudadri CS. Phacotrabeculectomy without mitomycin C in primary angle-closure and open-angle glaucoma. J Glaucoma. 2011. 20(1): 57-62.

[3] Seibold LK, Sherwood MB, Kahook MY. Wound modulation after filtration surgery. Surv Ophthalmol. 2012. 57(6): 530-50.

[4] Stahnke T, Löbler M, Kastner C, et al. Different fibroblast subpopulations of the eye: a therapeutic target to prevent postoperative fibrosis in glaucoma therapy. Exp Eye Res. 2012. 100: 88-97.

[5] Masoumpour MB, Nowroozzadeh MH, Razeghinejad MR. Current and Future Techniques in Wound Healing Modulation after Glaucoma Filtering Surgeries. Open Ophthalmol J. 2016. 10: 68-85.

[6] Reiter C, Wimmer S, Schultheiss A, Klink T, Grehn F, Geerling G. [Corneal epitheliopathy following trabeculectomy with postoperative adjunctive 5-fluorouracil]. Klin Monbl Augenheilkd. 2010. 227(11): 887-91.

[7] Fan GJC, Nguyen DQ, Soon AG, O'Connor J, Crowston JG. Wound Healing Modulation in Glaucoma Filtration Surgery- Conventional Practices and New Perspectives: Antivascular Endothelial Growth Factor and Novel Agents (Part II). J Curr Glaucoma Pract. 2014. 8(2): 46-53.

[8] Kiddee W, Orapiriyakul L, Kittigoonpaisan K, Tantisarasart T, Wangsupadilok B. Efficacy of Adjunctive Subconjunctival Bevacizumab on the Outcomes of Primary Trabeculectomy With Mitomycin C: A Prospective Randomized Placebo-controlled Trial. J Glaucoma. 2015. 24(8): 600-6.

[9] Greifner G, Roy S, Mermoud A. Results of CO2 Laser-assisted Deep Sclerectomy as Compared With Conventional Deep Sclerectomy. J Glaucoma. 2016. 25(7): e630-8.

[10] Elbably A, M OT, Mousa A, Elridy M, Badawy W, Elbably M. Deep Sclerectomy with Porous Collagen in Open-angle Glaucoma, Short-term Study. J Curr Glaucoma Pract. 2018. 12(2): 85-89.

[11] Jankowska-Szmul J, Dobrowolski D, Wylegala E. CO2 laser-assisted sclerectomy surgery compared with trabeculectomy in primary open-angle glaucoma and exfoliative glaucoma. A 1-year follow-up. Acta Ophthalmol. 2018. 96(5): e582-e591.

[12] Mermoud A. Sinusotomy and deep sclerectomy. Eye (Lond). 2000. 14 ( Pt 3B): 531-5. 
[13] Yu X, Chen C, Sun M, et al. CO2 Laser-assisted Deep Sclerectomy Combined With Phacoemulsification in Patients With Primary Open-angle Glaucoma and Cataract. J Glaucoma. 2018. 27(10): 906-909.

[14] Yao K, Wu R, Xu W, Chen P, Yin J. [Combined phacoemulsification, foldable intraocular lens implantation and trabeculectomy for cataract patients with glaucoma]. Zhonghua Yan Ke Za Zhi. 2000. 36(5): 330-3.

[15] Chen DZ, Koh V, Sng C, Aquino MC, Chew P. Complications and outcomes of primary phacotrabeculectomy with mitomycin C in a multi-ethnic asian population. PLoS One. 2015. 10(3): e0118852.

[16] Popovic V, Sjöstrand J. Long-term outcome following trabeculectomy: I Retrospective analysis of intraocular pressure regulation and cataract formation. Acta Ophthalmol (Copenh). 1991. 69(3): 299-304.

[17] Errico D, Scrimieri F, Riccardi R, Fedeli R, larossi G. Trabeculectomy with double low dose of mitomycin C - two years of follow-up. Clin Ophthalmol. 2011. 5: 1679-86.

[18] Landers J, Martin K, Sarkies N, Bourne R, Watson P. A twenty-year follow-up study of trabeculectomy: risk factors and outcomes. Ophthalmology. 2012. 119(4): 694-702.

[19] Bresson-Dumont H, Lehoux A, Baroux C, Foucher J, Santiago PY. [Clinical factors favoring excessive subconjunctival fibroblastic proliferation after glaucoma surgery]. J Fr Ophtalmol. 2008. 31(6 Pt 2): 2S747.

[20] Almatlouh A, Bach-Holm D, Kessel L. Steroids and nonsteroidal anti-inflammatory drugs in the postoperative regime after trabeculectomy - which provides the better outcome? A systematic review and meta-analysis. Acta Ophthalmol. 2018 .

[21] Wang B, Dong N, Xu B, Liu J, Xiao L. Efficacy and safety of intracameral triamcinolone acetonide to control postoperative inflammation after phacotrabeculectomy. J Cataract Refract Surg. 2013. 39(11): 1691-7.

[22] Gollakota S, Garudadri CS, Mohamed A, Senthil S. Intermediate Term Outcomes of Early Posttrabeculectomy Bleb Leaks Managed by Large Diameter Soft Bandage Contact Lens. J Glaucoma. 2017. 26(9): 816-821.

[23] Tseng VL, Kim CH, Romero PT, et al. Risk Factors and Long-Term Outcomes in Patients with Low Intraocular Pressure after Trabeculectomy. Ophthalmology. 2017. 124(10): 1457-1465.

[24] Olayanju JA, Hassan MB, Hodge DO, Khanna CL. Trabeculectomy-related complications in Olmsted County, Minnesota, 1985 through 2010. JAMA Ophthalmol. 2015. 133(5): 574-80. 
[25] Cutolo CA, Bagnis A, Scotto R, Bonzano C, Traverso CE. Prospective evaluation of CO2 laser-assisted sclerectomy surgery (CLASS) with Mitomycin C. Graefes Arch Clin Exp Ophthalmol. 2018. 256(1): 181186.

[26] Yick DW, Lee JW, Tsang S, Yeung BY, Yuen CY. Preliminary results of CO2 laser-assisted sclerectomy surgery (CLASS) in the treatment of advanced glaucoma in a Chinese population. Medicine (Baltimore). 2016. 95(45): e5294.

\section{Table}

Table 1. Baseline variables characteristics of between the groups.

\begin{tabular}{lccc}
\hline Variables & Study group $(\mathrm{N}=14)$ & Control group $(\mathrm{N}=16)$ & P-values \\
\hline Age (years) & $68.50 \pm 7.53$ & $70.81 \pm 6.55$ & 0.376 \\
Gender (female/male) & $6 / 8$ & $8 / 8$ & 0.696 \\
BCVA & $0.25 \pm 0.22$ & $0.22 \pm 0.19$ & 0.671 \\
IOP & $24.64 \pm 8.56$ & $23.19 \pm 8.45$ & 0.644 \\
Medications & 3 & 3 & 0.719 \\
\hline
\end{tabular}

BCVA, best corrected visual acuity; IOP, intraocular pressure; $\mathrm{P}<0.05$ at two tails was considered to be statistically significant.

\section{Figures}




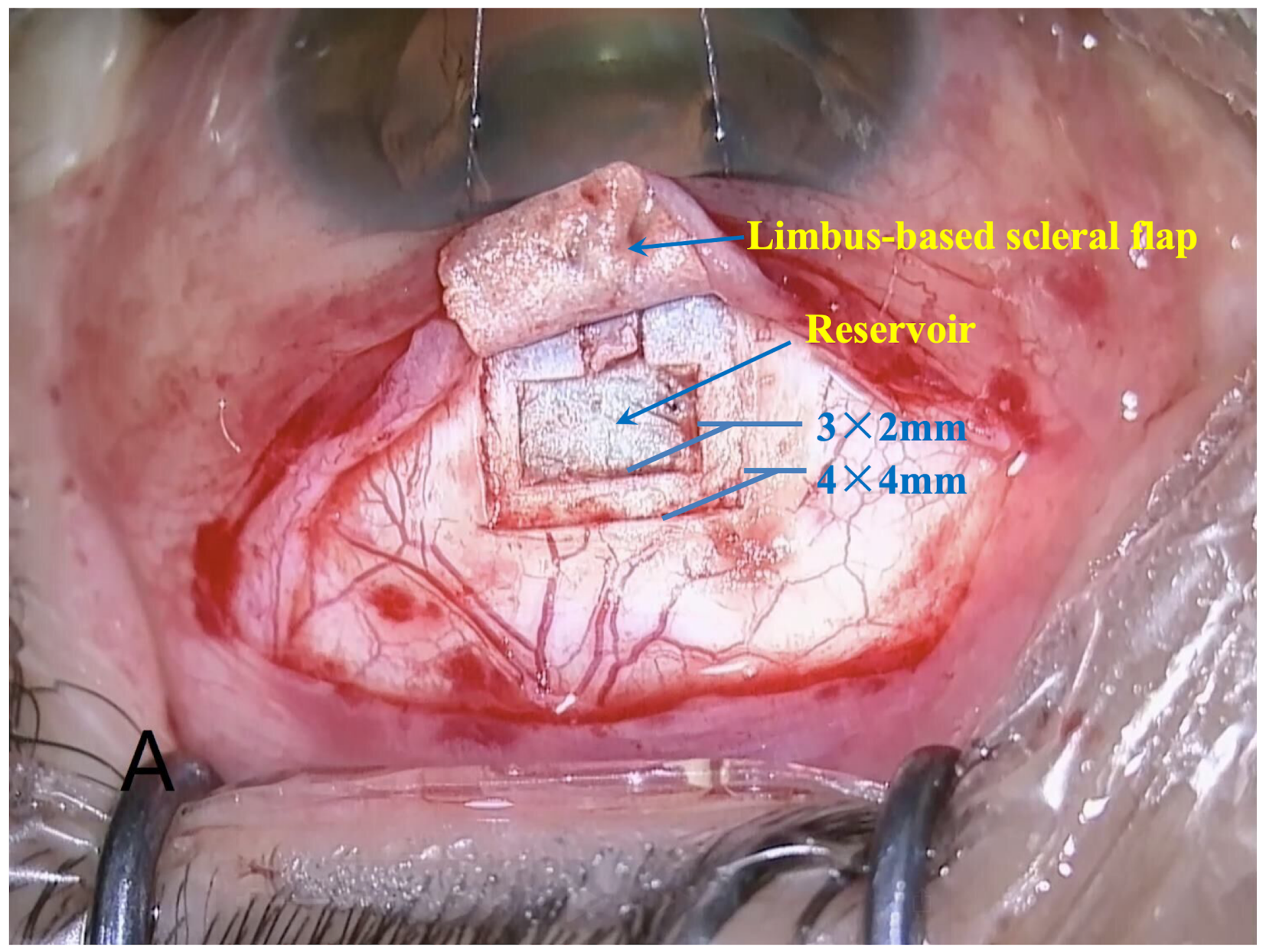

Figure 1.

\section{Figure 1}

Sketch map of SCPT surgery design. 


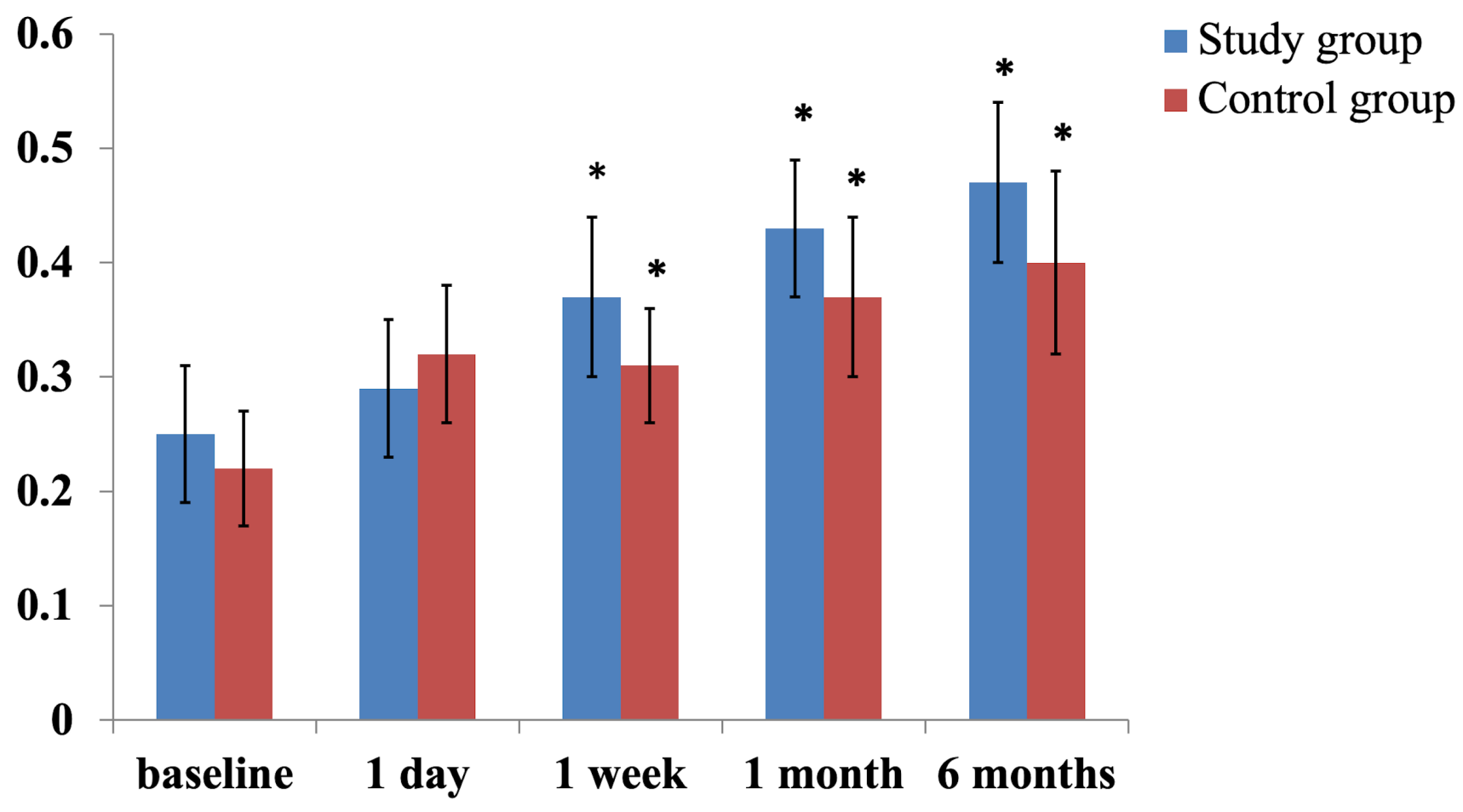

Figure 2.

Figure 2

Change in BCVA post operation in both groups. *: change in BCVA post operation was significantly different from baseline $(P<0.05)$. Nevertheless, the magnitude of visual improvement was comparable between both groups at each visit (all $P>0.05$ ). 


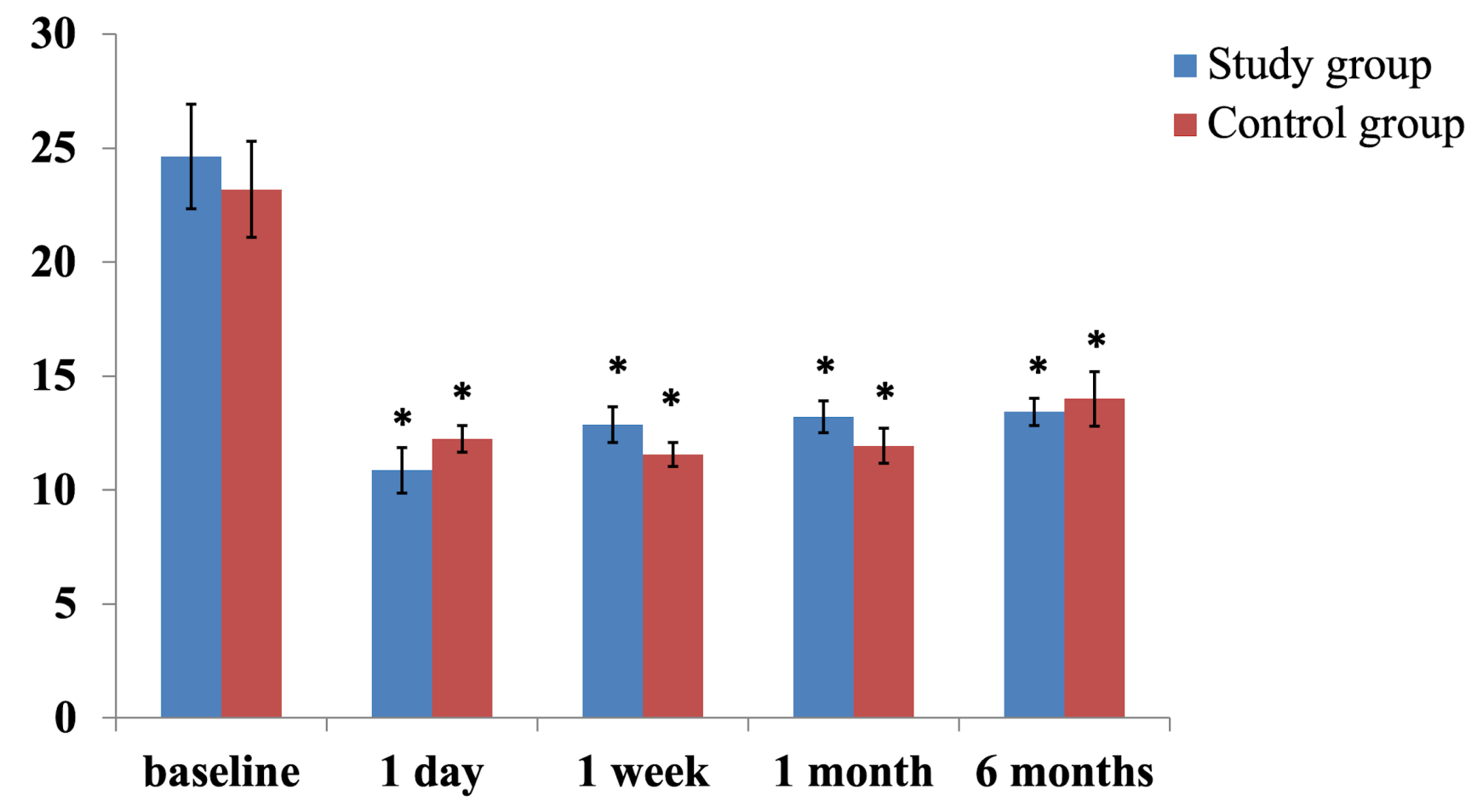

Figure 3.

Figure 3

Change in IOP post operation in both groups. *: change in IOP post operation was significantly different from baseline $(P<0.05)$. Nevertheless, there was no significant difference in the magnitude of IOP reduction between the 2 groups at the each visit (all $P>0.05$ ).

\section{Supplementary Files}

This is a list of supplementary files associated with this preprint. Click to download.

- Additionalsupportingfiles.xlsx 